\title{
Experimental supply equipment for active loop-based magnetic field mitigation system
}

\author{
J. C. del Pino López ${ }^{1}$, P. Cruz Romero ${ }^{2}$ \\ Department of Electrical Engineering, University of Seville \\ Camino de los Descubrimientos, s/n- 41092 Sevilla, Spain \\ ${ }^{1}$ phone: +34 54481282 - fax: +34 54487284 - e-mail: vaisat@esi.us.es \\ 22phone: +34 54481277 - fax: +34 54487284 - e-mail: plcruz@us.es
}

\begin{abstract}
Remarkable efforts have been devoted to research activities aiming at studying suitable techniques to achieve the desired reduction of the magnetic fields from transmission and distribution systems, with particular reference to high voltage overhead and buried lines. In this paper, an experimental DSPbased power source prototype designed to supply the precise currents to an active loop mitigation system located near the power line is analyzed. Its composition, current control technique and application on mitigating power lines magnetic field are described.
\end{abstract}

\section{Keywords}

Magnetic field mitigation, active loop, DSP, hysteresis current control.

\section{Introduction}

In the last decades, some epidemiological studies have suggested a weak association between exposure to power frequency magnetic fields and certain diseases. This is the reason why some local and central authorities have taken the initiative to develop a precautionary approach, with the purpose of reducing human exposure to magnetic fields produced by power lines and other electrical installations. These initiatives also included the adoption of exposure limits [1]. Due to these reasons, remarkable efforts have been devoted to research activities aiming at studying suitable techniques to achieve the desired reduction of the magnetic fields from transmission and distribution systems, with particular reference to high voltage overhead and buried lines. Various mitigation strategies are available and are generally applied and experienced at the design stage [2], but possibilities of achieving a real reduction are strongly reduced when installations are already in operation. One of the most attractive technologies applicable to operating power lines relies on the so-called active loop compensation, which consists on the design of antagonist sources capable of generating, in a given region, a magnetic field almost opposite to that of the original source.

In this paper, an experimental DSP-based power source prototype designed to supply the precise currents to an active loop mitigation system located near the power line is analyzed. Its composition, current control technique and application on mitigating power lines magnetic field are described.

\section{Shielding techniques}

From the environmental point of view, one of the most efficient methods to reduce the magnetic field from power overhead lines is using buried lines, but this technique may not be feasible due to the high costs, and even not enough in certain conditions; therefore, other methods have been proposed. The most extended ones are: magnetic field shielding with conductive or ferromagnetic materials and the use of passive loops. These are passive techniques based on the counteracting effect of an induced current that flows in shield conductors. In particular, compensation by passive loops consists on locating, near the power line, a conductor forming a loop, in such a way that the current induced in the loop generates a field that partially compensates the original field from the power line.

This passive technique can be improved if an external power source is provided to generate currents in the shield conductors, because it is possible to tune the amplitude and phase of these currents in order to increase the field reduction. This technique is known as active compensation, and considering magnetic field reduction in power lines, it is usually employed in a closed loop arrangement in the vicinity of the line. Such compensation can be achieved only partially; however a considerable reduction is generally possible at reasonable costs.

Due to these reasons, certain features in the supply system are required, in order to get the optimal choice of the currents to be driven to the compensation wires, so good reduction results could be achieved. To do this, some kind of control algorithm has to be implemented, based on measurements of the loop currents and magnetic field. So it is required to use a microcontroller to run the algorithm, and some signal adaptation circuits needed to adjust the required measurements. 


\section{Supply system description}

The experimental supply system proposed in this paper has been developed with the main aim of making smallscale laboratory experiences, in order to analyze active loop-based magnetic field mitigation systems behaviour. Figure 1 shows all the components developed and employed in the active loop supply system. It consists on the following devices:

- Half-bridge inverter.

- Mosfet driver circuit.

- Adaptation measurements circuit.

- DSP processor.

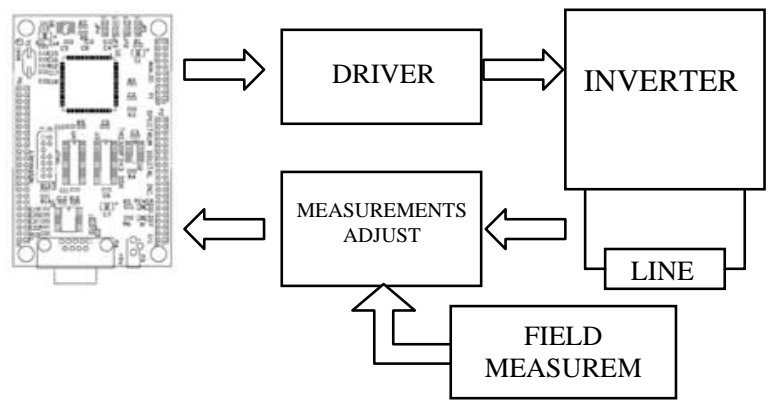

Fig. 1. System scheme.

In the experimental supply system considered a low-cost TMS320F243 DSP microcontroller has been used, delivered in a starter kit configuration. This is a high level microcontroller used in many different applications, from industrial components to office equipments. The main features of this device are:

- $\quad$ RAM and Flash memory.

- Analog and digital input/output channels.

- Integrated peripherals as A/D converter and event manager (PWM outputs, CAN module, etc.).

- Serial and Standard JTAG communication interface on board, to connect this device to a computer.

- Real time emulation to read signal measures from the PC.

This microcontroller is programmed by standard JTAG communication interface, and also permits to have a real time monitoring of the DSP's registers and signals in the computer. Some of these signals can be represented graphically in real time to see its evolution while system is running, thanks to the Code Composer software delivered with the DSP's Starter Kit.
To implement a control algorithm it is required that the microcontroller can acquire real time measures of the controlled system. This device uses its A/D converter inputs to do so, but it's necessary to adjust measures level to those supported by de DSP starter kit (0V to $5 \mathrm{~V})$. Due to this reason a measurements adaptation circuit was developed to adjust values taken form the magnetic field and the loop current (Fig. 1). Monitoring these magnitudes is done using a magnetic field sensor to measure the controlled field and a shunt resistance to acquire loop current.

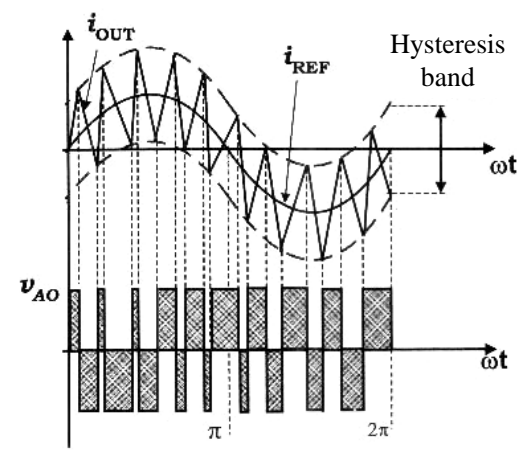

Fig. 2. Hysteresis current control algorithm.

As this is an experimental system, a simple hysteresis current control method was implemented to create the current flowing through the compensating loop (Fig. 2). With this algorithm it can be tuned the amplitude and phase of these currents, so a better field reduction can be achieved. In any case, thanks to the high features of the microcontroller, a more complex algorithm may be implemented later on.

Modelling compensating loop current resulting from the hysteresis control algorithm is done by a half bridge controlled inverter, developed to supply the current needed in the compensating loop at any moment. Its main components are MOSFET transistor.

The inverter configuration needs a DC voltage source to generate the loop current. In this case an external laboratory DC source was employed. The inverter works receiving orders from the hysteresis current control algorithm to change the state of the two MOSFETs of the bridge (Fig. 3). These orders are generated by the DSP's digital outputs, which are in the range of $0 \mathrm{~V}$ to $5 \mathrm{~V}$, and must be adjust to the range of $15 \mathrm{~V}$ to $+15 \mathrm{~V}$ needed to change the MOSFET state. To do so, a MOSFET driver circuit it's needed. In this case an IR2104 integrated circuit was used.

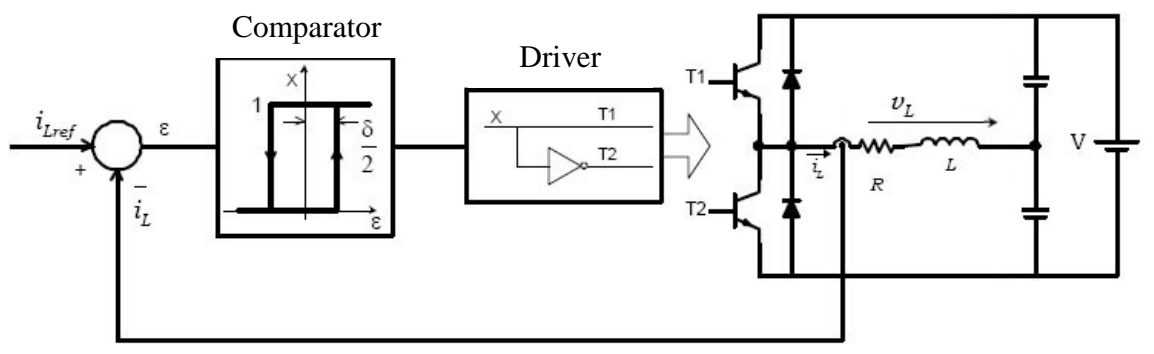

Fig. 3. System operation scheme. 
Additional components were necessary in order to improve system security, as fuses, heat radiator, etc. Figure 4 shows the complete experimental supply system developed.

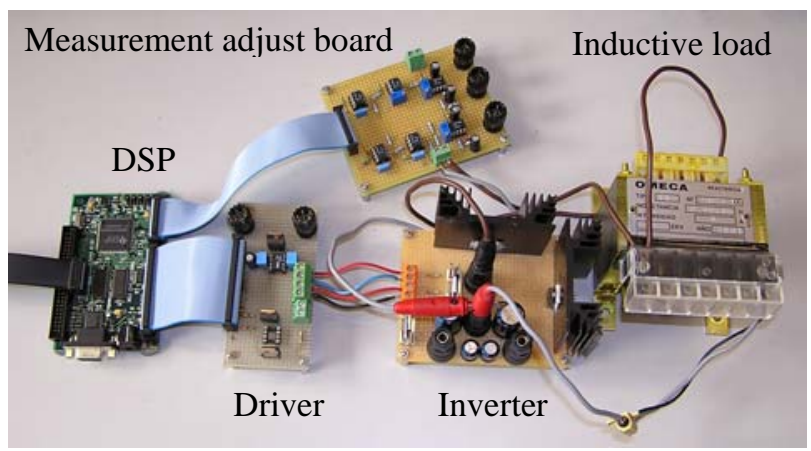

Fig. 4. Prototype of supply system.

\section{Experiences and results}

Before starting to compensate the magnetic field from a small-scale line, some computer simulations and laboratory experiences were realized to analyze the most important parameters that have influence on the current control algorithm, as hysteresis bandwidth, DC voltage applied to the half bridge inverter, resistance and loop inductance. This is important due to the influence of these parameters in the commutation frequency of the MOSFET transistors, that is limited by the DSP's processing speed. For example, it was concluded that higher values of the DC voltage applied to the inverter, lower loop inductance values or narrower hysteresis control bandwidth results in higher commutation frequency of the system. So it's necessary a previous tune up of the whole equipment before starting to mitigate.

Figure 5 shows an example of controlled current using an inductive load to simulate the loop inductance. It can be seen that the current wave is similar to those obtained in computer simulations.

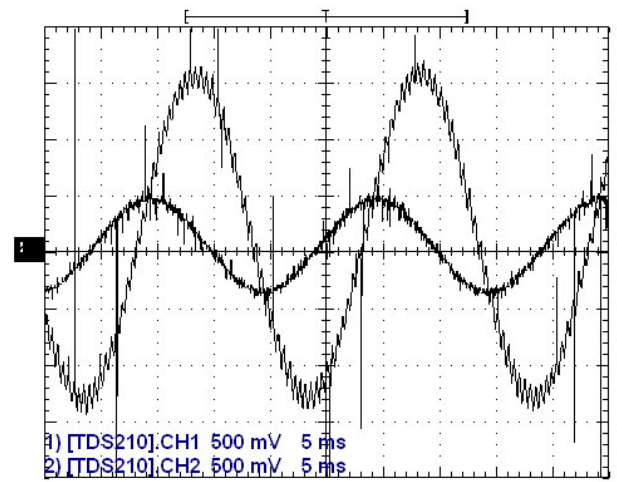

Fig. 5. Controlled loop current example.

Also, small-scale experiences were realized in a laboratory using an experimental line with a compensating loop arrangement (Fig. 6). The line was supplied with $220 \mathrm{~V}$ at $50 \mathrm{~Hz}$ from the network, but it was necessary to add some additional components to modify line current and ensure system's security, as transformers or variable resistors. Also an external inductance must be connected in series with the line to increase total inductance of the whole line, in order to achieve feasible values of commutation frequency in the experimental supply system, as mentioned before.

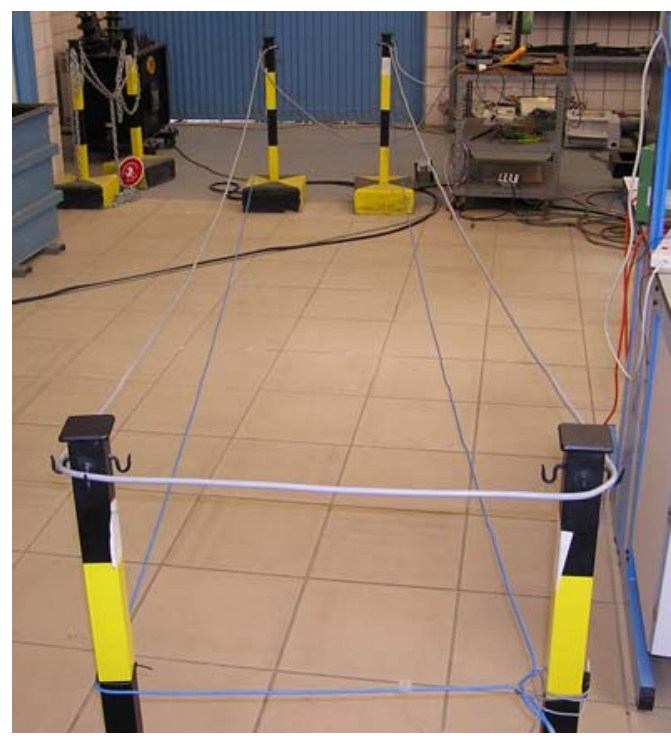

Fig. 6. Small-scale line and compensating loop.

A simple mitigating algorithm was developed in the DSP. In this case, a magnetic field sensor was employed to provide field measures to the microcontroller, in order to estimate power line current phase (detecting zero crossing) and its modulus, taking these values as a reference to generate the antagonist current through the compensating loop by the hysteresis control implemented.

Various experiences have been developed locating this sensor in different points along the experimental line, obtaining good mitigating results. Since studied configuration has been with the compensating loop just under the line, better mitigation results are obtained in the centre of the line, although field reduction in the external area is also achieved.

\section{Conclusion}

In this paper, an experimental supply equipment for an active loop-based magnetic field mitigation system is described. This prototype is the first step to develop a stand-alone system that will be used in the proximities of a power line, either an overhead or buried configuration.

Small-scale experiences in laboratory have been developed, analyzing those parameters that have important influence in the system in order to improve control algorithm, equipment performance and magnetic field reduction. So previous tune up of the whole equipment before starting to mitigate is needed. 
Several mitigation experiences have been developed locating compensating loop just under a small-scale test line, obtaining satisfactory results in the centre of the line and lower reduction in the external area.

Future experiences will be developed to optimize control algorithm and behaviour of the system. Also other compensating loop location will be considered, in order to achieve magnetic field reduction in areas far from the test line.

\section{Acknowledgement}

This project was supported by the Andalusian government under call "Financiación de un programa de actividades de transferencia de tecnología-2004” (Junta de Andalucia).

\section{References}

[1] National Radiological Protection Board, “Advice on Limiting Exposure to Electromagnetic Fields (0 300 GHz)”. Doc. NRPB 15 (2) mar. 2004 [Online].Available:http://www.hpa.org.uk/radiation/ publications/documents_of_nrpb/abstracts/absd152.htm.

[2] H. Olsson, P. Petterson and A. Eriksson, "Reduction of transmission line magnetic fields-possibilities and constraints”, Cigré Session 1990, paper 36-101.

[3] P. Cruz Romero, C. Izquierdo Mitchell, M. Burgos Payán, "Optimal design of active shielding for power lines”, 14th PSCC Sevilla 2002, Session 24, paper 2, page 1.

[4] P. L. Sergeant, L. R. Dupré, M. De Wulf and A. A. Melkebeek, "Optimizing active and passive Magnetic shields in induction heating by a genetic algorithm”,IEEE Trans. On Magnetics, Vol. 39, No. 6, November 2003. 